\title{
DETECTING UV RADIATION FROM NEARBY PULSARS WITH THE HUBBLE SPACE TELESCOPE
}

\author{
George G. Pavlov \\ A. F. Ioffe Institute of Physics and Technology
}

\begin{abstract}
Even old $\left(10^{6}\right.$ to $\left.10^{7} \mathrm{yr}\right)$ pulsars within a few hundred parsecs of the Sun should give UV and optical fluxes via thermal radiation or radiation from relativistic particles. The surface temperature of a neutron star depends on its mass, radius, magnetic field, and internal composition (existence of pion condensate, superfluidity of nucleons, etc.). If the temperature exceeds $\sim 2 \times 10^{4} \mathrm{~K}$, the thermal radiation can be detected by the Hubble Space Telescope. An analysis of the results will allow one to study the thermal evolution and inner structure of neutron stars in order to obtain additional constraints on pulsar models.
\end{abstract}

\section{Introduction}

A number of attempts by the Einstein Observatory and EXOSAT have been made recently to detect thermal radiation from neutron-star surfaces in the soft $\mathrm{X}$-ray range. Most of these have provided only upper limits on the neutronstar surface temperature $T_{\mathbf{s}}$ which lie between $\sim 2 \times 10^{5} \mathrm{~K}$ for the nearest pulsar PSR $1929+10$ (Alpar et al. 1987) and $2.5 \times 10^{6} \mathrm{~K}$ for the youngest, the Crab pulsar PSR 0531+21 (Harnden and Seward 1984). Positive (perhaps not very certain) results have been obtained for the Vela pulsar PSR 0833-45 $\left(8 \pm 2 \times 10^{5} \mathrm{~K}\right.$; Harnden et al. 1985), PSR $1509-58\left(\simeq 2.5 \times 10^{6} \mathrm{~K}\right.$; Seward and Harnden 1982), PSR 1055-52 $\left(\simeq 7 \times 10^{5} \mathrm{~K}\right.$; Cheng and Helfand 1983); Brinkmann and Ögelman 1987), and PSR 0656+14 $\left(4.5 \pm 1.5 \times 10^{5} \mathrm{~K}\right.$; Córdova et al. 1989).

After the successful launch of the Hubble Space Telescope (HST) there appears to be a new possibility of detecting the neutron-star surface radiation in the optical and/or UV range. A simple estimate shows that if a neutron star with radius $R=10 \mathrm{~km}$ and $T_{\mathrm{s}}=10^{5} \mathrm{~K}$ lies at a distance $d=100 \mathrm{pc}$, its magnitude in the $U$ band will be $m_{U}=26.2$ - that is, it can be detected by the HST. On the other hand, for $T_{\mathrm{s}}=10^{6} \mathrm{~K}$ and $d=1 \mathrm{kpc}$, one has $m_{\mathrm{U}}=$ 30. This means that the nearby old pulsars may be more promising for detecting the surface UV radiation than more distant young pulsars.

In order to choose the exposure time, some preliminary estimates of probable $T_{\mathbf{s}}$ values are desirable. The Einstein Observatory survey gave upper limits on the $\mathrm{X}$-ray luminosity in the range $0.5-5 \times 10^{30} \mathrm{ergs} \mathrm{s}^{-1}$ for 10 pulsars within $500 \mathrm{pc}$ from the Sun (Helfand 1983). The corresponding surface temperature limits are $T_{\mathrm{s}} \simeq 1.5-3.5 \times 10^{5} \mathrm{~K}$ for $R=7-12 \mathrm{~km}$. The predictions of current theo- ries of neutron-star cooling (see e.g. Tsuruta 1985, Nomoto and Tsuruta 1987, Shibazaki and Lamb 1989, Yakovlev et al. 1990) are very uncertain; $T_{\mathrm{s}} \sim 10^{3}$ to $6 \times 10^{5} \mathrm{~K}$ for the typical ages of the nearby pulsars $t \sim 10^{6}$ to $10^{7} \mathrm{yr}$. The wide scatter of the theoretical predictions results mainly from different assumptions about the properties of superdense matter in neutron-star interiors (equation of state, the appearance of nucleon superfluidity, effects of the magnetic field, etc.). The most probable estimates of the surface temperature seem to lie in the range $T_{\mathrm{s}} \sim 10^{4}$ to $10^{5} \mathrm{~K}$.

\section{Proposal and perspectives for the HST}

The nearest pulsars (see table 1 for the indicated temperatures) can be detected by the Faint $\mathrm{Ob}$ ject Camera (FOC/48) available on the HST. In the standard 512 $\times 512$-pixel format, it has a field of view $22^{\prime \prime} \times 22^{\prime \prime}$ with a pixel size $0^{\prime \prime} .043 \times 0^{\prime \prime} .043$, whereas the image radius for a point source is $\simeq 0^{\prime \prime} .1-0^{\prime \prime} .2$. Since pulsar positions are known with very high precision $\left(\simeq 0^{\prime \prime} .1-0^{\prime \prime} .2\right)$, blind acquisition is appropriate (its accuracy may be $\simeq 1^{\prime \prime}-2^{\prime \prime}$ ). The largest signal-to-noise ratio $S / N$ can be reached with the F180LP long pass filter $(\lambda \lambda=1800-6000 \AA)$ which cuts off the airglow background from the Ly $\alpha$ line at $1216 \AA$ and $O$ I triplet at $1304 \AA$. This allows one to detect the thermal radiation from the nearest pulsars $(S / N>5)$ during a 30-40 min exposure if the surface temperatures exceed $2-3 \times 10^{4} \mathrm{~K}$, which is an order of magnitude lower than the limits obtained in the X-ray region. Other filters can be used to get additional spectral information for evaluating the temperature more accurately. The table gives the expected $S / N$ ratios for several filters $(\lambda \lambda=3050-6000 \AA$ for the F305LP, and 3020- 
$3730 \AA$ for the $F 342 W$ ), assuming some probable temperatures $3-5 \times 10^{4} \mathrm{~K}$. The spectral information can also be used to insure a more reliable identification of the neutron star among the other objects which may appear in field of view at low galactic latitudes $b$. This is especially important just for the nearest pulsar, PSR 1929+10, which lies at $b=-3.9^{\circ}$. The other two pulsars lie at high latitudes, $b=43.7^{\circ}$ and $69.2^{\circ}$ and are expected to be the only objects at their specified positions. The identification could be strictly confirmed in future observations, preferably with other filters, with allowance for the large proper motion of the pulsars (up to $0.37 \mathrm{arcsec} / \mathrm{yr}$ for PSR $1133+16$ ).

Table 1 The nearest pulsars

\begin{tabular}{|lccc|}
\hline PSR & $1929+10$ & $0950+08$ & $1133+16$ \\
\hline$d, \mathrm{pc}$ & 80 & 90 & 150 \\
$t, 10^{6} \mathrm{yr}$ & 3 & 18 & 5 \\
$\mu, \operatorname{arcsec} / \mathrm{yr}$ & 0.088 & 0.034 & 0.371 \\
$b$ & -3.9 & $43: 7$ & 6992 \\
$P, \mathrm{~s}$ & 0.23 & 0.25 & 1.19 \\
$B, 10^{12} \mathrm{G}$ & 0.5 & 0.3 & 2.1 \\
Assumed $T_{\mathrm{s}}, \mathrm{K}$ & 30000 & 30000 & 50000 \\
$t_{\text {exp }}, \min ; \mathrm{S} / \mathrm{N}$ & & & \\
F180LP filter & $38 ; 7$ & $36 ; 5$ & $40 ; 5$ \\
F305LP filter & $38 ; 4$ & & \\
F342W filter & $38 ; 3$ & & \\
\hline
\end{tabular}

A program of observations has now been accepted by the Telescope Allocation Committee. The exposure times in table 1 correspond to the total allocated spacecraft time of 4 hours. Successful realization of the program will permit us to 'view' neutron stars for the first time, to evaluate their temperatures, and to obtain information on their thermal evolution. The latter is important for studying neutron-star physics and the properties of very dense matter. Most significant is the equation of state of nuclear matter which is now poorly known. For instance, according to Van Riper and Lamb (1981), an old neutron star $\left(t=3 \times 10^{6} \mathrm{yr}\right)$ with $M=1.4 \mathrm{M}_{\odot}$ will have $T_{\mathrm{s}}=10^{5} \mathrm{~K}$ for a soft (BPS) equation of state and $T_{3}=7 \times 10^{3} \mathrm{~K}$ for a hard (PS) equation of state, provided the internal composition is standard (no pion condensates, no quark matter); in the non-standard case the same star can be much cooler, e.g., $T_{s} \simeq 3-4 \times 10^{4} \mathrm{~K}$ for a soft equation of state. The surface temperature also depends on the neutron-star mass. An example presented by Tsuruta (1979) shows that a neutron star of age $10^{6} \mathrm{yr}$ may have $T_{\mathrm{s}} \simeq 2 \times 10^{4}$,
$2 \times 10^{3}$ and $2 \times 10^{5} \mathrm{~K}$ if its mass equals $1.4,0.5$ and $0.1 \mathrm{M}_{\odot}$, respectively. Also important are the effects of neutron and proton superfluidity in the neutronstar inner crust and central core. They reduce $T_{\mathbf{3}}$ for $t \gtrsim 10^{6} \mathrm{yr}$ (by a factor of $\gtrsim 2$ for $t=3 \times 10^{6} \mathrm{yr}$ in examples given by Nomoto and Tsuruta 1987). On the other hand, in neutron stars that are being spun down by external torques, the superfluid is expected to be rotating faster than the rest of the star. Dissipation of this differential rotation (due to pinning of neutron superfluid vortices in the inner crust) may yield significant additional heating (Shibazaki and Lamb 1989). In particular, for a 'standard' cooling model of a neutron star with $M=1.4 \mathrm{M}_{\odot}, R=15.8 \mathrm{~km}$ at $t=3 \times 10^{6} \mathrm{yr}$, $T_{\mathrm{s}}$ equals $1.4 \times 10^{5}$ and $4.3 \times 10^{5} \mathrm{~K}$ if $\mathrm{J}=3 \times 10^{43}$ and $3 \times 10^{45} \mathrm{~g} \mathrm{~cm}^{2} \mathrm{rad} \mathrm{s}^{-1}$, respectively. Here $J$ is the total differential angular momentum of the frictionally coupled superfluid layers. Finally, the surface temperature depends on magnetic field effects. On the one hand, a strong magnetic field may reduce the crust opacity which speeds up the cooling and reduces $T_{\mathrm{s}}$ (see, e.g., Van Riper and Lamb 1981). On the other hand, the ohmic dissipation of the internal field may significantly heat the core and delay the cooling at $t \gtrsim 10^{6} \mathrm{yr}$ (Yakovlev, Urpin, and Haensel 1990). Since the theoretical predictions for $T_{s}(t)$ are clearly very model-dependent, measuring $T_{3}$ for pulsars of different ages would help to select more appropriate models.

If we succeeded in detecting the nearby pulsars in Phase I of the HST observations, an extension of the program (Phase II) might be proposed. Based on the Phase I results, one might observe the pulsars with other filters to get new information about their spectra and probably the chemical abundance at the neutron-star surface (by detecting a difference in the irradiated spectrum from the blackbody one). It would also be useful to perform a timing analysis of the surface radiation: the UV radiation may be modulated at the pulsar period if the surface is heated inhomogeneously. Polarization measurements can also be of interest since the surface magnetic field polarizes the radiation. Finally, the program can be extended to other nearby pulsarse.g., the nearest pulsars

PSR 0809+74 $\left(d=170 \mathrm{pc}, t=1 \times 10^{8} \mathrm{yr}\right)$, PSR $1952+29\left(d=200 \mathrm{pc}, t=3 \times 10^{9} \mathrm{yr}\right)$, PSR 1451-68 $\left(d=230 \mathrm{pc}, t=4 \times 10^{7} \mathrm{yr}\right)$, and the more distant but younger

PSR 0656+14 $\left(d=400 \mathrm{pc}, t=1 \times 10^{5} \mathrm{yr}\right)$.

Acknowledgments: The author is grateful to Yu. N. Gnedin, I. I. Kumkova, M. A. Pogodin and D. G. Yakovlev for useful discussions. 